PROCEEDINGS OF THE

AMERICAN MATHEMATICAL SOCIETY

Volume 130, Number 6, Pages 1597-1602

S 0002-9939(01)06238-4

Article electronically published on December 20, 2001

\title{
HINDMAN SPACES
}

\author{
MENACHEM KOJMAN
}

(Communicated by Alan Dow)

\begin{abstract}
A topological space $X$ is Hindman if for every sequence $\left(x_{n}\right)_{n}$ in $X$ there exists an infinite $D \subseteq \mathbb{N}$ so that the sequence $\left(x_{n}\right)_{n \in F S(D)}$, indexed by all finite sums over $D$, is IP-converging in $X$. Not all sequentially compact spaces are Hindman. The product of two Hindman spaces is Hindman.

Furstenberg and Weiss proved that all compact metric spaces are Hindman. We show that every Hausdorff space $X$ that satisfies the following condition is Hindman:

(*) The closure of every countable set in $X$ is compact and first-countable.

Consequently, there exist nonmetrizable and noncompact Hindman spaces. The following is a particular consequence of the main result: every bounded sequence of monotone (not necessarily continuous) real functions on $[0,1]$ has an IP-converging subsequences.
\end{abstract}

\section{INTRODUCTION}

Let us start by recalling Hindman's finite-sum theorem about partitions of $\mathbb{N}$. Let $\mathbb{N}=\{1,2, \ldots\}$ denote the set of natural numbers, with the convention that $n=\{0,1, \ldots, n-1\}$.

Definition 1. A set $A$ of natural numbers is called an IP set if there exists an infinite set $D \subseteq \mathbb{N}$ so that $F S(D) \subseteq A$, where $F S(D)=\left\{\sum \alpha: \alpha \subseteq D\right.$ and $\alpha \neq \emptyset$ is finite $\}$.

Theorem 2 (Hindman [8]). If the integers are partitioned into finitely many parts, then one of the parts is IP. In fact, if an IP set is partitioned into finitely many parts, then one of the parts is IP.

In this paper we examine which topological spaces satisfy a topological generalization of Hindman's theorem. We shall call those spaces Hindman spaces.

The results show that in addition to the compact metric spaces, which were shown to be Hindman in [4] (see also Theorem 8.14 in [5]), there are plenty of Hindman spaces which are neither compact nor metrizable.

Received by the editors October 2, 2000 and, in revised form, December 20, 2000.

1991 Mathematics Subject Classification. Primary 05C55, 54F65; Secondary 04A20, 11P99, $26 \mathrm{~A} 40$.

Key words and phrases. Hindman's theorem, converging sequence, compactification, finite sums, nonmetrizable topological spaces.

(C)2001 American Mathematical Society 


\section{HiNDMAN SPACES}

Hindman's theorem can be formulated topologically as follows: every sequence $\left(x_{n}\right)$ in a finite (Hausdorff) space $X$ has a converging subsequence whose set of indices is IP. It is tempting to define a Hindman space as a Hausdorff space $X$ that satisfies this property, but that definition would not get us very far. The blame lies on the distributive law.

Theorem 3. If a $T_{1}$ space $X$ satisfies that for every sequence $\left(x_{n}\right)_{n \in \mathbb{N}}$ there exists a converging subsequence $\left(x_{n}\right)_{n \in A}$ for some IP set $A$, then $X$ is finite.

Proof. For every $n \in \mathbb{N}$ let $i(n)=\max \left\{i: 2^{i} \mid n\right\}$. Note that $i\left(n_{1}+n_{2}\right)>i\left(n_{1}\right)$ if $i\left(n_{1}\right)=i\left(n_{2}\right)$ and that $i\left(n_{1}+n_{2}\right)=i\left(n_{1}\right)$ if $i\left(n_{1}\right)<i\left(n_{2}\right)$.

Suppose $X$ satisfies the condition of the theorem and that $X$ is infinite. Necessarily, $X$ contains a converging injective sequence $\left(x_{n}\right)_{n \in \mathbb{N}}$. Define a new sequence $\left(y_{n}\right)_{n}$ in $X$ by $y_{n}:=x_{i(n)}$.

Suppose now that $D \subseteq \mathbb{N}$ is infinite and $A=F S(D)$ satisfies that $\left(y_{n}\right)_{n \in A}$ is converging. Assume first that $\left\{y_{n}: n \in A\right\}$ is finite. Then, since $X$ is $T_{1}$, $\left(y_{n}\right)_{n \in A}$ is eventually constant. Pick $n_{1}<n_{2}$ in $D$ with $y_{n_{1}}=y_{n_{2}}=y_{n_{1}+n_{2}}$. Then $i\left(n_{1}\right)=i\left(n_{2}\right)=i\left(n_{1}+n_{2}\right)$, a contradiction.

Thus, one must have that $\left\{y_{n}: n \in A\right\}$ is infinite. In this case we may assume, by thinning $D$ out, that $i\left(n_{1}\right)<i\left(n_{2}\right)$ for all $n_{1}<n_{2}$ in $D$. But then $y_{n_{1}+n_{2}}=y_{n_{1}}$ for all $n_{1}<n_{2}$ in $D$, and $\left(y_{n}\right)_{n \in A}$ cannot converge.

To obtain a meaningful topological definition of a Hindman space we use the notion of IP-convergence, introduced by Furstenberg and Weiss in [4] (and presented in detail also in [5], Chapter 8).

Definition 4. (1) Suppose that $D \subseteq \mathbb{N}$ is infinite. An IP-sequence in a topological space is a sequence indexed by $F S(D)$ for some infinite $D \subseteq \mathbb{N}$. An IP-sequence $\left(x_{n}\right)_{n \in F S(D)}$ in a topological space $X I P$-converges to a point $x \in X$ if for every neighborhood $u$ of $x$ there exists $m \in \mathbb{N}$ so that $\left\{x_{n}: n \in F S(D \backslash m)\right\} \subseteq u$.

(2) A topological space $X$ is Hindman if for every sequence $\left(x_{n}\right)$ in $X$ there exists an infinite set $D \subseteq \mathbb{N}$ so that $\left(x_{n}\right)_{n \in F S(D)}$ IP-converges to some $x \in X$.

Suppose that $\left(x_{n}\right)_{n \in F S(D)}$ is IP-converging in $X$. For every infinite $D_{1} \subseteq D$, $\left(x_{n}\right)_{n \in F S\left(D_{1}\right)}$ is clearly also IP-converging to $x$. By thinning any infinite $D$ out and enumerating it increasingly as $\left\{d_{1}, d_{2}, \ldots\right\}$, we may assume that for every $n \in F S(D)$ there is a unique finite $\alpha \subseteq \mathbb{N}$ for which $n=\sum_{i \in \alpha} d_{i}$ and denote that unique $\alpha$ by $\alpha_{D}(n)$. Call an infinite $D \subseteq \mathbb{N}$ for which $\alpha_{D}(n)$ is defined for all $n \in F S(D)$ a sparse subset of $\mathbb{N}$.

Let $E=\left\{2^{i}: i \in \mathbb{N}\right\}$. Then $F S(E)=\mathbb{N}$ and $\alpha_{E}(n)$ is defined for all $n$, and is exactly the set of $i$ 's for which the $i$-th digit in the binary expansion of $n$ is equal to 1 .

Let $x_{n}=n$ for all $n$. The IP-sequence $\left(x_{n}\right)_{n \in F S(E)}$ IP-converges to 0 in the 2-adic topology on $\mathbb{N} \cup\{0\}$, (that is, in the metric topology generated by the metric $d(m, n)=1 / \max \{i: i \mid(m-n)\}$ for $m \neq n)$. Let $D_{1}=\left\{3^{i}: i \in \mathbb{N}\right\}$. Now $F S\left(D_{1}\right) \subseteq F S(E)=\mathbb{N}$ but $\left(x_{n}\right)_{n \in F S\left(D_{1}\right)}$ does not IP-converge to 0 . The possibility just sketched, of a non-IP-converging IP-sequence contained in an IP-converging IPsequence, needs to be handled in proving that the product of two Hindman spaces is Hindman. 
Definition 5. Suppose $D$ is sparse. $D_{1} \subseteq F S(D)$ is normal in $F S(D)$ if $D_{1}$ is sparse and for all $n_{1}, n_{2} \in D_{1}, n_{1} \neq n_{2} \Rightarrow \alpha_{D}\left(n_{1}\right) \cap \alpha_{D}\left(n_{2}\right)=\emptyset$.

Fact 6. If $D_{1}$ is normal in $F S(D)$ then:

(1) $F S\left(D_{1}\right) \subseteq F S(D)$.

(2) Whenever $\left(x_{n}\right)_{n \in F S(D)} I P$-converges to $x$ in some space $X,\left(x_{n}\right)_{n \in F S\left(D_{1}\right)}$ also IP-converges to $x$.

Proof. The first part is clear. For the second part, let $u$ be an arbitrary neighborhood of $x$ and fix $m$ such that $x \in F S(D \backslash m) \Rightarrow x_{n} \in u$. Since $\left\{a_{D}(n): n \in D_{1}\right\}$ is a disjoint family, there is some $m^{\prime}$ such that $n \in D_{1} \backslash m^{\prime} \Rightarrow\left\{d_{i}: i \in \alpha_{D}(n)\right\}>m$.

Lemma 7. Suppose that $D$ is sparse. Then there exists $D_{1} \subseteq F S(D)$ which is normal both in $F S(E)$ and in $F S(D)$.

Proof. For finite sets of integers $\alpha_{1}, \alpha_{2}$ we write $\alpha_{1}<\alpha_{2}$ to mean $\max \alpha_{1}<\min \alpha_{2}$. By induction on $i$ find a sequence of integers $n_{i} \in F S(D)$ such that $i<j \Rightarrow$ $\alpha_{D}\left(n_{i}\right)<\alpha_{D}\left(n_{j}\right) \wedge \alpha_{E}\left(n_{i}\right)<\alpha_{E}\left(n_{j}\right)$. Let $n_{1}=\min D$. Suppose $n_{i}$ has been chosen. Choose some $m_{i}$ so that $2^{m_{i}}>\sum_{\ell \leq i} n_{\ell}$ and fix $T \subseteq D \backslash 2^{m_{i}}$ with $|T|=2^{m_{i}}$. It is easy to see that for some nonempty subset $T_{1} \subseteq T$ the sum $\sum T_{1}$ is divisible by $2^{m_{i}}$. Let $n_{i+1}=\sum T_{1}$. It is clear that $\alpha_{D}\left(n_{i+1}\right)>\alpha_{D}\left(n_{i}\right)$ and, since $2^{m_{i}} \mid n_{i+1}$, $\alpha_{E}\left(n_{i+1}\right)>\alpha_{E}\left(n_{i}\right)$. Let $D_{1}=\left\{n_{i}: i \in \mathbb{N}\right\}$.

Lemma 8. If $X$ is Hindman, then for every sparse $D \subseteq \mathbb{N}$ and an IP-sequence $\left(x_{n}\right)_{n \in F S(D)}$ there exists $D_{1}$ normal in $F S(D)$ such that $\left(x_{n}\right)_{n \in F S\left(D_{1}\right)}$ IP-converges to some $x \in X$.

Proof. Suppose $\left(x_{n}\right)_{n \in F S(D)}$ is given in some Hindman space $X$ where $D$ is sparse. Let $\left\{d_{i}: i \in \mathbb{N}\right\}$ be the increasing enumeration of $D$. Define $g: \mathbb{N} \rightarrow F S(D)$ by $g\left(\sum_{i \in \alpha} 2^{i}\right)=\sum_{i \in \alpha} d_{i}$ for all nonempty finite $\alpha \subseteq \mathbb{N}$. Since $X$ is Hindman, there exists a sparse $F \subseteq \mathbb{N}$ so that $\left(x_{g(n)}\right)_{n \in F S(F)}$ IP-converges to some $x \in X$. Using the previous lemma, we may assume that $F$ is normal in $F S(E)$. This implies that $D_{1}:=g[F]$ is normal in $F S(D)$ and that $\left(x_{n}\right)_{n \in F S\left(D_{1}\right)}$ IP-converges to $x$.

Fact 9. The product of two Hindman spaces is Hindman.

Proof. Suppose that $Z=X \times Y$ where $X, Y$ are Hindman and suppose that $\left(x_{n}, y_{n}\right)_{n}$ is a sequence in $Z$. Since $X$ is Hindman, one can fix a sparse $D \subseteq \mathbb{N}$ so that $\left(x_{n}\right)_{n \in F S(D)}$ IP-converges to some $x \in X$. By Lemma 8 there is some $D_{1}$ normal in $F S(D)$ so that $\left(y_{n}\right)_{n \in F S(D)}$ IP-converges to some $y \in Y$. Now $\left(x_{n}, y_{n}\right)_{n \in F S\left(D_{1}\right)}$ IP-converges to $(x, y)$ in $Z$.

Every Hindman space is surely sequentially compact. The following shows that being Hindman is strictly stronger than being sequentially compact.

Theorem 10. There exists a Hausdorff, compact, sequentially compact, separable space which is first-countable at all points but one, which is not Hindman.

Proof. Let $\mathcal{F}$ be a maximal element in $\{\mathcal{A}: \mathcal{A}$ is an infinite family of infinite almost disjoint subsets of $\mathbb{N}$ and every member of $\mathcal{A}$ is not IP $\}$. Then $\mathcal{F}$ is in fact a maximal almost disjoint family (suppose to the contrary that $B \subseteq \mathbb{N}$ is infinite and almost disjoint to every member in $A$. By thinning out, $B$ can be made non-IP).

For every $A \in \mathcal{F}$ let $p_{A} \notin \mathbb{N}$ be a distinct point. Define a topology $\mathcal{T}$ on $Y=\mathbb{N} \cup\left\{p_{A}: A \in \mathcal{F}\right\}$ by requiring that $B \in \mathcal{T}$ if and only if for every $p_{A} \in B$ 
the set $A \backslash B$ is finite. $\mathcal{T}$ is a Hausdorff topology on $Y$ with respect to which $\mathbb{N}$ is a discrete subspace. Since $\left\{p_{A}\right\} \cup A$ is a compact neighborhood of $p_{A}$ for every $A \in \mathcal{F}, \mathcal{T}$ is locally compact.

Let $X=Y \cup\{p\}$ be the one-point compactification of $Y$. Let us check that $X$ is sequentially compact. Let $\left(x_{n}\right)$ be an arbitrary sequence in $X$. If the range of the sequence is finite, there is little to prove. If the range of the sequence is infinite, assume, by passing to a subsequence, that the sequence is injective and that its range is contained either in $\mathbb{N}$ or in $\left\{p_{A}: A \in \mathcal{F}\right\}$. In the first case, the maximality of $\mathcal{F}$ guarantees the existence of some $A \in \mathcal{F}$ and a subsequence $\left(x_{n_{k}}\right)$ whose range is contained in $A$; this subsequence converges to $p_{A}$. In the other case, the sequence itself converges to $p$, since the intersection of its range with every compact subset of $Y$ is finite.

To see that $X$ is not Hindman, let $\left(x_{n}\right)_{n}$ in $X$ be defined by $x_{n}=n$. For each $p_{A},\left\{p_{A}\right\} \cup A$ is a neighborhood of $p_{A}$ which does not contain any IP subsets of $\mathbb{N}$. Therefore, for no $D \subseteq \mathbb{N}$ can $\left(x_{n}\right)_{n \in F S(D)}$ IP-converge to $p_{A}$. To see that for no $D$ can $\left(x_{n}\right)_{n \in F S(D)}$ IP-converge to $p$, let $D$ be arbitrary, and consider the family of sets $\{F S(D \backslash m): m \in \mathbb{N}\}$. Every finite intersection of members from this (countable) family is infinite, hence there exists an infinite pseudo-intersection of the family, namely an infinite set $Z$ so that for every $m \in \mathbb{N}$ satisfies $Z \backslash F S(D \backslash m)$ is finite. By maximality of $\mathcal{F}$, there is some member $A \in \mathcal{F}$ for which $A \cap Z$ is infinite. The set $X \backslash\left(\left\{p_{A}\right\} \cup A\right)$ is an open neighborhood of $p$, but for every $m \in \mathbb{N}$, the intersection $F S(D \backslash m) \cap A$ is infinite; therefore, $\left(x_{n}\right)_{n \in F S(D)}$ does not IP-converge to $p$.

Theorem 8.14 in [5], due to Furstenberg and Weiss [4, implies that compact metric spaces are Hindman. The following theorem extends this result, using the technology of idempotents in $(\beta \mathbb{N},+) . \beta \mathbb{N}$ is the set of all ultrafilters over $\mathbb{N}$ and the operation of addition in $\mathbb{N}$ can be extended to $\beta \mathbb{N}$ (turning it to a left continuous and compact semi-group) via the formula

$$
A \in U+V \Longleftrightarrow\{n: A-n \in U\} \in V .
$$

For a good and recent exposition of this material see [7] (with the caution that they reverse the operation from the way it is described in (1D)). An ultrafilter $U \in \beta \mathbb{N}$ is an idempotent if $U+U=U$. The Galvin-Glazer proof of Hindman's theorem by using an idempotent in $(\beta \mathbb{N},+)$ is presented also in [6] and in [2].

Theorem 11. Suppose that a Hausdorff space $X$ satisfies the following condition:

(*) The closure of every countable set in $X$ is compact and first-countable.

Then $X$ is Hindman.

Proof. Suppose $\left(x_{n}\right)$ is given in $X$. Let $D=\operatorname{cl}_{X}\left\{x_{n}: n \in \mathbb{N}\right\}$. Fix an idempotent $U \in \beta \mathbb{N}$ and let $U^{\prime}$ be the ultrafilter over $D$ which is defined by $A \in U^{\prime} \Longleftrightarrow$ $\left\{n: x_{n} \in A\right\} \in U$. Since $D$ is compact, we can find $x \in D$ so that every open neighborhood of $x$ (in $D$ ) belongs to $U^{\prime}$. Fix a decreasing neighborhood base $\left(u_{n}\right)_{n}$ at $x$ with $u_{1}=D$, and denote $\bar{u}_{n}=\left\{k \in \mathbb{N}: x_{k} \in u_{n}\right\}$. We shall find an infinite $\left\{m_{n}: n \in \mathbb{N}\right\} \subseteq \mathbb{N}$ so that for every finite nonempty $\alpha \subseteq \mathbb{N}, \sum_{n \in \alpha} m_{n} \in \bar{u}_{\min \alpha}$. This clearly implies IP-convergence of $\left(x_{m}\right)_{m \in F S\left(\left\{m_{n}: n \in \mathbb{N}\right\}\right)}$ to $x$.

By induction on $n$ define a set $A_{n} \subseteq \mathbb{N}$ and a number $m_{n}$ that satisfy for all $n$ :

(1) $A_{n} \in U$,

(2) $A_{n} \subseteq \bar{u}_{n}$, 
(3) $m_{n} \in A_{n}$ and $m_{n}>\sum_{i<n} m_{i}$,

(4) $k \in A_{n+1} \Rightarrow\left\{k, k+m_{n}\right\} \subseteq A_{n}$.

Let $A_{1}=A$. Suppose that $A_{n}$ and $m_{1}, \ldots m_{n-1}$ are defined and $A_{n}$ satisfies conditions (1) and (2). We shall now define $m_{n}$ which satisfies (3) and then define $A_{n+1}$ so that (4) is satisfied and so that (1) and (2) hold for $n+1$.

$A_{n} \in U$ and $U$ is an idempotent (hence nonprincipal). So $B_{n+1}=\left\{k: A_{n}-k \in\right.$ $U\} \cap A_{n} \in U$. Fix $m_{n} \in B_{n+1}$ which satisfies $m_{n}>\sum_{i<n} m_{i}$. Condition (3) is now satisfied. Let $A_{n+1}=A_{n} \cap\left(A_{n}-m_{n}\right) \cap \bar{u}_{n+1}$. Now condition (4) is satisfied. Since $A_{n+1}$ is defined as the intersection of three sets in $U, A_{n+1} \in U$, so condition (1) holds for $n+1$. Since $A_{n+1} \subseteq \bar{u}_{n+1}$, condition (2) for $n+1$ holds also. That completes the inductive definition.

To show that for each nonempty finite set $\alpha \subseteq N$, it holds that $\sum_{n \in \alpha} m_{n} \in$ $\bar{u}_{\min \alpha}$, we argue by induction on $|\alpha|$ that $\sum_{n \in \alpha} m_{n} \in A_{n}$, which suffices, since $A_{n} \subseteq \bar{u}_{n}$ by condition (2). If $\alpha=\{n\}$ is a singleton, then $\sum_{n \in \alpha} m_{n}=m_{n} \in A_{n}$ by condition (3). Suppose $\alpha=\left\{m_{n_{1}}, m_{n_{2}}, \ldots, m_{n_{t}}\right\}$. By the induction hypothesis, $k:=\sum_{i=2}^{t} m_{n_{i}} \in A_{n_{2}}$. But $A_{n_{2}} \subseteq A_{m_{1}+1}$ (since the $A_{n}$ 's are decreasing by (4)), so by condition (4), $m_{n_{1}}+k \in A_{m_{1}}$.

Every space which satisfies condition $(*)$ is Hindman. The class of spaces which satisfies $(*)$ includes all compact metric spaces and all compact linearly ordered topological spaces. In particular:

Corollary 12. For every sequence $\left(\alpha_{n}\right)$ of ordinal numbers there is an IP-converging sequence $\left(\alpha_{n}\right)_{n \in F S(D)}$ for some infinite $D \subseteq \mathbb{N}$.

The space $\omega_{2}$ with the order topology is an example of a space which satisfies $(*)$ which is neither first-countable nor compact (although all of its separable subspaces are metrizable). Helly's space of all monotone functions from $[0,1]$ to $[0,1]$ with the topology induced from the product topology on $[0,1]^{[0,1]}$ (see [12]) is an example of a separable, compact and first-countable space which, therefore, satisfies $(*)$ which is not metrizable. The instance of Theorem 11 for this particular space gives:

Corollary 13. Suppose that $f_{n}:[0,1] \rightarrow[0,1]$ is monotone for each $n$. Then there is a monotone function $f:[0,1] \rightarrow[0,1]$ and an infinite $D \subseteq \mathbb{N}$ so that $\left(f_{n}\right)_{n \in F S(D)}$ IP-converges pointwise to $f$.

\section{REFERENCES}

[1] J. E. Baumgartner, A short proof of Hindman's Theorem, J. Combinatorial Theory A, 17:384386, 1974. MR 50:6873

[2] V. Bergelson, Ergodic Ramsey Theory - an update, Ergodic theory and $Z^{d}$-actions. London Math. Soc. Lecture Note Series 228, 1-61, 1996. MR 98g:28017

[3] V. Bergelson and N. Hindman, Nonmetrizable topological dynamics and Ramsey Theory, Trans. AMS, 320:293-320, 1990. MR 90k:03046

[4] H. Furstenberg, and B. Weiss, Topological dynamics and combinatorial number theory, $J$. Analyse Math. 34:61-85, 1978. MR 80g:05009

[5] H. Furstenberg, Recurrence in Ergodic Theory and Combinatorial Number Theory, Princeton University Press, Princeton, NJ, 1981. MR 82j:28010

[6] Ronald L. Graham, Bruce L. Rothschild and Joel H. Spencer, Ramsey Theory, John Wiley E Sons, 1990. MR 90m:05003

[7] N. Hindman and D. Strauss. Algebra in the Stone-Čech compactification, de Gruyter, Berlin, 1998. MR 99j:54001

[8] N. Hindman, Finite sums from sequences within cells of partitions of $\mathbb{N}, J$. Combinatorial Theory A, 17:119-184, 1974. MR 50:2067 
[9] N. Hindman, Ultrafilters and combinatorial number theory, Number Theory Carbondale, M. Nathanson, ed., Lecture Notes in Math., 751, 1979. MR 81m:10019

[10] N. Hindman, Partitions and sums and products of integers, Trans. AMS, 247, 227-245, 1979. MR 80b:10022

[11] M. Kojman, Van der Waerden spaces, Proc. AMS, to appear.

[12] Lynn Arthur Steen and J. Arthur Seebach, Jr., Counterexamples in topology, SpringerVerlag, New York, 1978. MR 80a:54001

[13] B. L. van der Waerden, Beweis eine Baudetschen Vermutung Nieus Arch. Wisk., 15:212-216, 1927.

Department of Mathematics, Ben Gurion University of the Negev, Beer-Sheva, 84105, ISRAEL

E-mail address: kojman@cs.bgu.ac.il 